This item was submitted to Loughborough's Research Repository by the author.

Items in Figshare are protected by copyright, with all rights reserved, unless otherwise indicated.

\title{
Incorporating driver preferences Into eco-driving assistance systems using optimal control
}

PLEASE CITE THE PUBLISHED VERSION

https://doi.org/10.1109/tits.2020.2977882

\section{PUBLISHER}

Institute of Electrical and Electronics Engineers (IEEE)

\section{VERSION}

AM (Accepted Manuscript)

\section{PUBLISHER STATEMENT}

Personal use of this material is permitted. Permission from IEEE must be obtained for all other uses, in any current or future media, including reprinting/republishing this material for advertising or promotional purposes, creating new collective works, for resale or redistribution to servers or lists, or reuse of any copyrighted component of this work in other works.

\section{LICENCE}

\section{All Rights Reserved}

\section{REPOSITORY RECORD}

Fleming, James, Xingda Yan, and Roberto Lot. 2020. "Incorporating Driver Preferences into Eco-driving Assistance Systems Using Optimal Control”. Loughborough University.

https://hdl.handle.net/2134/12623813.v1. 


\title{
Incorporating driver preferences into eco-driving assistance systems using optimal control
}

\author{
James Fleming*, Xingda Yan ${ }^{\dagger}$, Roberto Lot $^{\ddagger}$ \\ ${ }^{*}$ Wolfson School of Mechanical, Electrical and Manufacturing Engineering, Loughborough University, UK \\ ${ }^{\dagger}$ Department of Mechanical Engineering Sciences, University of Surrey, UK \\ ${ }^{\ddagger}$ Department of Industrial Engineering, University of Padova, Italy
}

\begin{abstract}
Recently there have been several proposals for 'ecodriving assistance systems', designed to save fuel or electrical power by encouraging behaviours such as gentle acceleration and coasting to a stop. These systems use optimal control to find driving behaviour that minimises vehicle energy losses. In this paper, we introduce a methodology to account for driver preferences on acceleration, braking, following distances and cornering speed in such eco-driving optimal control problems. This consists of an optimal control model of acceleration and braking behaviour containing several physically-meaningful parameters to describe driver preferences. If used in combination with a model of fuel or energy consumption, this can provide an adjustable trade-off between satisfying those preferences and minimising energy losses. We demonstrate that the model gives comparable performance to existing car-following and cornering models when predicting drivers' speed in these situations by comparison with real-world driving data. Finally, we present an example highway braking scenario for an electric vehicle, illustrating a trade-off between satisfying driver preferences on vehicle speed and acceleration and reducing electrical energy usage by up to $43 \%$.
\end{abstract}

\section{INTRODUCTION}

Drivers' acceleration and braking behaviour has a significant effect on vehicle fuel consumption, a fact that was first recognised over four decades ago [1]. Reduction of fuel consumption, and therefore of $\mathrm{CO}_{2}$ emissions due to transport, has become a topical issue in recent years due to concerns about climate change [2]. Economical driving behaviour, or 'eco-driving', has been suggested as a method which can reduce $\mathrm{CO}_{2}$ emissions from road vehicles by $10 \%$ with current technology by encouraging drivers to accelerate gently, to anticipate signals and traffic flow to avoid stops, to maintain an even speed, and to avoid idling [3]. This view has been reinforced by recent results of the naturalistic driving study UDRIVE, which suggest that braking behaviour, gear shifting and the velocity choice on the motorway have effects on fuel consumption of greater than $10 \%$ [4].

Training programs have been designed to encourage ecodriving behaviors with positive results [5], but after these programs many drivers revert back to their original driving styles over time [6]. A further complication for eco-driving is that avoiding stops at intersections requires prediction of traffic flow and signal changes, which is difficult for humans. Introducing coasting behaviour before corners and junctions is known to reduce fuel consumption, but coasting effectively requires prior knowledge of the timing of signal changes and accurate models of vehicle fuel consumption and dynamics [7]. A potential remedy to both of these problems is to provide active feedback to the driver on their behaviour, for instance by using auditory, visual or haptic human-machine interfaces (HMIs) within a vehicle [8], [9]. There is considerable research interest in improving these interfaces using methods such as V2X communication [10] or machine learning [11].

To put the problem of minimising fuel consumption in a mathematical framework, the eco-driving problem can be formulated as an optimal control problem [12], [13]. This requires modelling of the vehicle powertrain, losses due to aerodynamic drag and rolling resistance, and modelling of gear-shifting, leading to a complex mixed-integer nonlinear programming problem [14] or a dynamic programming problem [15]. Although effective, these methods require large computational effort to compute the optimal velocity profile. Model predictive control (MPC) has been suggested as a computationally tractable alternative to solve the problem during driving [16]. If available, the incorporation of fuel consumption maps and knowledge of road grade to such predictive optimisations can considerably improve performance [17]. Recently, researchers have also considered the effect of road curvature on ecodriving [18], where constraints on lateral acceleration cause the vehicle to reduce speed in curves.

User acceptance is a well-known issue with existing automotive ADAS, for example for collision-warning systems [19] that rely on knowledge of typical vehicle-following behaviour. Based on the large variations between drivers observed in naturalistic studies, one suggestion to improve user acceptance of ADAS is to make the system adaptive, adjusting to the driver by estimating parameters representing their driving style in real-time [20]. For eco-driving assistance systems this possibility has already been explored for the specific scenario of approaching intersections [21]. Several simplified models of car-following behaviour have already been developed for use in traffic simulations, such as the Intelligent Driver Model (IDM) which uses a differential equation for velocity to model the motion of a vehicle with acceleration limits and a preferred velocity and following distance [22]. For curve driving behaviour, models of tolerable lateral accelerations while cornering have appeared in the human factors literature, such as the model due to Reymond and co-workers appearing in [23]. From the point of view of adapting system parameters, real-time adaptation of the IDM has been demonstrated [24] and the authors have recently developed an automated fitting 
method for models of cornering speeds [25].

In this paper, we present a general method of accounting for the effects of driver following behaviour and cornering speed choice in an eco-driving assistance system. By considering the modelling of longitudinal driver behaviour in an optimal control framework, it is possible to trade-off the objectives and preferences of the driver with the objective of energyefficiency. This provides a tuning parameter that can give behaviour intermediate between the two extremes of economical driving and natural driving, and gives a systematic way to account for driving styles in an eco-driving assistance system.

To be able to represent a variety of drivers who have different preferences with regard to acceleration, braking, and following distances, our driver model contains a small number of parameters that may readily be estimated from naturalistic driving data. To simplify this process we choose the set of model parameters to match those of the IDM, for which parameter fitting procedures are well known. We present a methodology based on an optimal control formulation of the IDM and subsequent quadratic approximation near equilibrium points that allows us to give physical meaning to the otherwisearbitrary weighting parameters appearing in the cost function. This leads to a model that gives similar behaviour to the IDM in car-following scenarios, but that can additionally handle position-dependent constraints on velocity resulting from cornering and is suitable for incorporating into an ecodriving optimal control problem. We validate this model by comparing its predictions with real-world driving data and existing models of cornering and car-following.

Because the new model of driver behaviour is based on optimal control in which the driver chooses the minimum possible value of a cost function, we may interpret the cost as a measure of a driver's 'dissatisfaction' with a particular vehicle trajectory and control history. Under this interpretation the model yields the acceleration profile that is most satisfying to the driver in terms of speed, acceleration, and vehicle spacing. Accordingly, we refer to it as the 'Driver Satisfaction Model' (DSM) to easily contrast it with, for example, the IDM.

\section{LITERATURE REVIEW}

\section{A. Optimal control}

Optimal control is an extension of the calculus of variations which considers how to choose the input of a dynamical system to optimise some performance criterion. The field originated with the work of Bellman [26] and Pontrygin [27] and was initially applied to problems in aeronautics and spaceflight, but later found application in many other fields. The optimal control problems we consider have the form

$$
\begin{array}{rl}
\min _{\mathbf{u}(t) \in \mathcal{U}} & J[\mathbf{x}, \mathbf{u}] \\
\text { s.t. } & \dot{\mathbf{x}}=f(\mathbf{x}, \mathbf{u}, t) \\
& g(\mathbf{x}, \mathbf{u}, t) \leq 0 \\
& \mathbf{x}(0)=\mathbf{x}_{0}, \quad \mathbf{x}(T) \in \mathcal{X}_{T}
\end{array}
$$

where $J[\mathbf{x}, \mathbf{u}]$ is the cost function given by:

$$
J[\mathbf{x}, \mathbf{u}]=\int_{0}^{T} L(\mathbf{x}, \mathbf{u}, t) \mathrm{dt}+\phi(\mathbf{x}(T))
$$

In this cost function, $L(\mathbf{x}, \mathbf{u}, t)$ and $\phi(\mathbf{x}(T))$ are referred to as the stage cost and terminal cost respectively. The vector $\mathbf{x} \in \mathbb{R}^{n}$ represents the state of a dynamical system, which evolves according to the differential equation $\dot{\mathbf{x}}=f(\mathbf{x}, \mathbf{u}, t)$, and the vector $\mathbf{u} \in \mathbb{R}^{m}$ represents a control input. The cost function $J$ models a quantity that should be kept small in a particular application, such as fuel usage, elapsed time, or deviation from a reference.

The problem of eco-driving can be formulated in an optimal control framework by choosing the stage cost $L(\mathbf{x}, \mathbf{u}, t)$ to be the rate of fuel consumption of the vehicle [13]. However, choosing to minimise fuel consumption alone can lead to behaviour that is unnatural for a human driver, such as travelling far below the speed limit or leaving large spacings to the preceding vehicle. To address this, the authors of the present paper considered a modified optimal control problem in [28] in which the cost function has the form

$$
J=\int_{T_{i}}^{T_{f}}\left(L_{d}+\alpha L_{f}\right) \mathrm{dt}
$$

where $L_{f}$ is a fuel consumption term, and the additional term $L_{d}$ represents driver preferences on speed, acceleration, and inter-vehicle spacings. By adjusting the weighting parameter $\alpha$ between $\infty$ and 0 , it is possible to obtain behaviour that is intermediate between minimising fuel consumption and fully respecting the driver's preferred driving style. This gives a parameter that can be tuned to ensure that the solution of the optimal control problem does not seem unnatural to the driver, respecting normal following distances for example.

Analytical solutions of optimal control problems only exist in special cases, such as when there are bounds on the input and both cost and dynamics are linear [29] or for unconstrained linear systems with quadratic costs [30]. However, the latter case is practically useful because systems may often be linearised and cost functions locally approximated as quadratic, an approach that we adopt in the current work to relate the weighting parameters of our model's cost function to the parameters of existing models of driver behaviour. In practice, optimal control problems are often solved using numerical methods. Two effective approaches are multiple shooting, in which the input is parameterised as a function of time and the state integrated piecewise over times of interest [31], and direct collocation, in which both the input and state are incorporated into a nonlinear programming problem as decision variables [32]. These methods of solution are not guaranteed to find the global solution of the problem, yet work well in practice.

\section{B. Models of vehicle following}

Many models of driver acceleration and braking behaviour express the acceleration of a individual driver as a function of their current speed and the relative position and velocity of the preceding vehicle. This leads to a differential equation describing motion known as a 'car-following' model. Such carfollowing models have their origin in [33], which proposes to describe vehicle-following by the delay differential equation

$$
\dot{v}=\lambda\left[v_{L}(t-\tau)-v(t-\tau)\right],
$$


in which $v$ denotes ego-vehicle velocity, $v_{L}$ denotes the velocity of the leader, $\tau$ is a time-delay parameter and $\lambda$ is a sensitivity parameter. That is, the driver accelerates in proportion to the relative velocity of the vehicle ahead, incorporating some time delay. This shows a good fit to experimental data, but is an oversimplified view of actual driver behaviour as there is no consideration of the distance to the lead vehicle. Subsequent works have incorporated this distance, as well as other factors, leading to many car-following models [22], [34], [35], [36]. Because of the large number of models available in the literature, in the remainder of this section we restrict our attention to two that are directly relevent to the present work. For a more extensive overview, we direct the reader to the review which may be found in [37].

The IDM was proposed in [22] to model congested states of traffic, and is a car-following model described by the ordinary differential equation

$$
\dot{v}=a\left[1-\left(\frac{v}{v_{d}}\right)^{\delta}-\left(\frac{s^{*}(v, \Delta v)}{x_{L}-x}\right)^{2}\right]
$$

where the desired inter-vehicle spacing $s^{*}(v, \Delta v)$ is given by

$$
s^{*}(v, \Delta v)=s_{0}+T v+\frac{v \Delta v}{2 \sqrt{a b}}
$$

and where $x_{L}$ denotes the position of the preceding vehicle and $\Delta v=v_{L}-v$ is the relative velocity of that vehicle. The model parameters $a, b, v_{d}, s_{0}, T$ may vary between drivers and are respectively the maximum acceleration, desired deceleration, free-flow velocity, minimum desired spacing, and the desired time-gap to the leading vehicle. Our interest in this model is that it provides a useful set of parameters that characterise driver behaviour during car-following, which have well-defined physical meanings and for which there exist welltested fitting procedures [38].

A car-following model using linear quadratic optimal control, and hence that is related to the model developed in the present paper, appeared in [39]. This considered the driver as an optimal controller that regulates the vehicle spacing and velocity to desired set points $s^{*}$ and $v^{*}$ by minimising

$$
J=\int_{0}^{\infty}\left[q_{s}\left(s-s^{*}\right)^{2}+q_{v}\left(v-v^{*}\right)^{2}+\dot{v}^{2}\right] \mathrm{dt}
$$

which, as there are no acceleration constraints, leads to linear driver behaviour according to the differential equation:

$$
\dot{v}=-k_{v}\left(v-v^{*}\right)-k_{s}\left(s-s^{*}\right)
$$

Although appealing due to its interpretation that the driver attempts to achieve preferred velocities and spacings to a lead vehicle, the model is unrealistic at large distances as then the acceleration is large and unbounded. A further practical issue is that the parameters $q_{s}$ and $q_{v}$ in the cost function have no physical interpretation, and instead the model must be fit to a particular driver by estimating $k_{s}$ and $k_{v}$ from driving data.

\section{Models of cornering speed}

A model of driver speed choice in curves was suggested in [40] which, inspired by models of braking based on timeto-collision (TTC), considered a quantity called time-to-lanecrossing (TLC) to explain variations in driving speed for corners of different curvatures. By removing visual feedback while entering a corner, the authors demonstrated that drivers estimate the curvature of a corner on approach and make an anticipatory steering action based on the perceived curvature. Later work using a driving simulator also demonstrated that differences between individuals' cornering speeds could be explained by differences in their steering competance [41], measured as their ability to accurately track the centre of a lane on a straight road.

Further study of driver speed in curves was carried out by Reymond et al. in [23], which investigated the effect of lateral acceleration in drivers' choice of speed when negotiating curves, demonstrating that drivers choose their cornering speed based on a lateral acceleration limit and a 'safety margin' of error when estimating curvature. Denoting road curvature as $\kappa$, this leads to a model of maximum driver speed in the curve given by

$$
v_{\max }=\sqrt{\frac{\Gamma_{\max }}{\kappa+\Delta}}
$$

where the parameters $\Gamma_{\max }$ and $\Delta$ represent the maximum lateral acceleration and the curvature safety margin. These parameters vary from driver to driver, and together characterise a particular driver's cornering speed preferences. As it lacks a name, we refer to this model as 'Reymond's model' for the remainder of the paper.

A similar curvature-speed relationship is suggested in [42], where the maximum allowable speed in curves is predicted as:

$$
v_{\max }=\theta \kappa^{-\frac{1}{3}}
$$

An interesting feature of that work is that a trajectory for vehicle longitudinal motion is generated by minimising a cost function that penalises time and includes a quadratic penalty on jerk, with this velocity limit included as a constraint. Hence, this models not only the choice of speed for a given curvature, but also the transition while entering and exiting the corner. This is especially relevant to eco-driving applications, since coasting down to reduce speed before cornering can significantly affect fuel consumption.

\section{The Driver SATISFACtion Model}

We now describe the Driver Satisfaction Model (DSM) as a model of driver acceleration behaviour, noting that our goal is a model that gives similar behaviour to the IDM in car-following situations, but that can also handle velocity constraints due to cornering and be incorporated into ecodriving optimal control problems. In the following sections, we introduce a stage cost function for the DSM and then relate its weighting parameters to the parameters of the IDM. Variations in speed due to cornering are then incorporated by adding a constraint on vehicle velocity, in a similar manner to [42].

\section{A. Development of cost function and constraints}

The stage cost function used in the DSM is based on the optimal control model of car-following given in [39], which has the cost function (6). Using this as a starting point, we make modifications to add a limit to the maximum acceleration 
and to modify the penalty on inter-vehicle spacing to better reflect the driver's indifference to far-away vehicles.

When the spacing $s$ between vehicles is large, the behaviour of (6) becomes dominated by the $s-s^{*}$ term. This is quite unrealistic, as drivers are likely to be indifferent to far away vehicles. To address this, we introduce a penalty function for vehicle spacing $s$ that tends to a constant value when $s$ is large, reflecting this indifference. Hence we use a penalty function that is approximately quadratic near its minimum, but is a rational function with a quadratic term in the denominator according to

$$
\psi_{s}(s)=\frac{\left(s / s_{d}-1\right)^{2}}{\left(s / s_{d}\right)^{2}+1}
$$

in which $s_{d}$ denotes the driver's 'desired' spacing. The justification for referring to $s_{d}$ as the driver's desired spacing is that it corresponds to the minimum of the penalty, and therefore will correspond to an equilibrium value for the model. The penalty is approximately constant for $s \gg s_{d}$, so that in this case the driver behaviour is unaffected by any lead vehicle.

To complete the cost, we add a quadratic penalty function for velocity that penalises the difference to a 'desired' velocity which would be chosen by the driver in the absence of a lead vehicle. This is similar to (6). Combining these penalty functions then leads to the DSM stage cost

$$
L_{\mathrm{dsm}}=(u / a)^{2}+\beta\left(v / v_{d}-1\right)^{2}+\gamma \frac{\left(s / s_{d}-1\right)^{2}}{\left(s / s_{d}\right)^{2}+1}
$$

where $\beta$ and $\gamma$ represent weighting parameters to trade-off the three competing objectives.

Noting that minimisation of (11) alone may lead to unrealistically large accelerations when either $s-s^{*}$ or $v-v^{*}$ are large in magnitude, we also introduce a limit on the maximum acceleration. Considering the acceleration as a control input and denoting it by $u$, we limit it so that

$$
u \leq a
$$

where $a$ denotes a constant parameter which may be considered as a characteristic of a particular driver. We introduce a similar constraint on the vehicle spacing, specifying that

$$
s \geq 0
$$

at all times in order to avoid collisions with the lead vehicle.

\section{B. Choice of weighting parameters}

At this point, the parameters $s_{d}, \beta$ and $\gamma$ are still arbitrary, making the model difficult to apply. Given the extensive literature on parameter estimation for car-following models, we relate these values to the parameters of the IDM. The following result provides a variational formulation of the IDM, and will be used throughout the remainder of this section.

Theorem 1. For fixed initial velocity $v(0)=v_{i}$, the IDM (4) is optimal for the stage cost

$$
L_{i d m}=\left[\left(\frac{v}{v_{d}}\right)^{\delta}+\left(\frac{s^{*}}{s}\right)^{2}-1\right]^{2}+\left(\frac{u}{a}\right)^{2}-\left(\frac{u}{a}\right)\left(\frac{s^{*}}{s}\right)^{2}
$$

and, writing $v_{f}=v(T)$, the terminal cost

$$
\phi_{i d m}\left(v_{f}\right)=\frac{2 v_{f}}{a(\delta+1)}\left[\left(\frac{v_{f}}{v_{d}}\right)^{\delta}-\delta-1\right]
$$

subject to the constraints $\dot{v}=u, \dot{s}=v-v_{L}$.

Proof. From (4), the IDM is optimal for the stage cost

$$
L_{1}=\left[\frac{\dot{v}}{a}+\left(\frac{v}{v_{d}}\right)^{\delta}+\left(\frac{s^{*}}{s}\right)^{2}-1\right]^{2}
$$

as this has a minimum value of zero which is attained if and only if (4) is satisfied at all times of interest.

Expanding, collecting terms in $\dot{v} / a$, and integrating from $t=0$ to $t=T$, we find,

$$
\int_{0}^{T} L_{1} \mathrm{dt}=\int_{0}^{T} L_{\mathrm{idm}} \mathrm{dt}+\int_{0}^{T} 2\left[\left(\frac{v}{v_{o}}\right)^{\delta}-1\right] \frac{\dot{v}}{a} \mathrm{dt}
$$

whereby the second term can be integrated by changing variables from $t$ to $v$ :

$$
\int_{v_{i}}^{v_{f}} \frac{2}{a}\left[\left(\frac{v}{v_{o}}\right)^{\delta}-1\right] \mathrm{dv}=\phi_{\mathrm{idm}}\left(v_{f}\right)-\phi_{\mathrm{idm}}\left(v_{i}\right)
$$

Because the initial velocity is fixed, the term $\phi_{\text {idm }}\left(v_{i}\right)$ is constant and may be dropped from the cost function without affecting the solution.

The cost function (14) is convenient for analysis, but not for direct minimisation via numerical methods. In particular the first and second derivatives are equal to zero when $v=0$ and approach zero as $s \rightarrow \infty$, which typically causes convergence problems. It also has a singularity at $s=0$, which leads to ill-conditioning when $s$ is small.

We construct expressions for $s_{d}, \beta$ and $\gamma$ in terms of the parameters in the IDM by relating the two stage costs (11) and (14) when considering small perturbations from equilibrium values. We first consider a vehicle on a straight road clear of other traffic. In that case, we may take $s \rightarrow \infty$ in the IDM stage cost (14):

$$
L_{\mathrm{idm}} \rightarrow\left[\left(v / v_{d}\right)^{\delta}-1\right]^{2}+(u / a)^{2}
$$

To fix $\beta$ such that we obtain similar behaviour to (14) for $v$ near $v_{d}$, we find a quadratic approximation $\tilde{q}(v)$ of

$$
q(v)=\left[\left(v / v_{d}\right)^{\delta}-1\right]^{2}
$$

by expansion in a Taylor series about $v_{d}$ :

$$
\tilde{q}(v)=q\left(v_{d}\right)+q^{\prime}\left(v_{d}\right)\left(v-v_{d}\right)+\frac{1}{2} q^{\prime \prime}\left(v_{d}\right)\left(v-v_{d}\right)^{2}
$$

Noting that $q\left(v_{d}\right)=q^{\prime}\left(v_{d}\right)=0$, we have

$$
\tilde{q}(v)=\frac{\delta^{2}}{v_{d}^{2}}\left(v-v_{d}\right)^{2}
$$

after evaluating $q^{\prime \prime}\left(v_{d}\right)$. Comparing to the quadratic velocity penalty in the DSM stage cost (11), this suggests choosing

$$
\beta=\delta^{2}
$$


to obtain similar behaviour near equilibrium when $s$ is large. In particular, with this choice of $\beta$ we have

$$
\lim _{s \rightarrow \infty} \frac{\partial^{2} L_{\mathrm{dsm}}}{\partial v^{2}} \approx \lim _{s \rightarrow \infty} \frac{\partial^{2} L_{\mathrm{idm}}}{\partial v^{2}}
$$

considering second derivatives at the equilibrium point $v=v_{d}$.

To find an expression for $s_{d}$, we consider the special case where there is a lead vehicle with a constant velocity $v_{d}$. Under these conditions, the IDM has an equilibrium at:

$$
v_{\mathrm{eq}}=v_{L}, \quad s_{\mathrm{eq}}=\frac{s_{0}+T v_{L}}{\sqrt{1-\left(v_{L} / v_{d}\right)^{\delta}}}
$$

By inspection of (11) we see that the minimum over $s$, and hence the equilibrium spacing when the lead vehicle is travelling at constant velocity, is at $s=s_{d}$. We would like this to correspond to the equilibrium of the IDM, so we choose:

$$
s_{d}(v)=\frac{s_{0}+T v}{\sqrt{1-\left(v_{L} / v_{d}\right)^{\delta}}}
$$

This gives greater following distances for larger values of $v$, in common with the IDM and many other models. The dependence on $v$ rather than $v_{L}$ is deliberate and leads to behaviour that is more similar to the IDM in braking, for which we recall $s^{*}=s_{0}+T v$.

To fix $\gamma$, we once again consider taking a local approximation of the IDM stage cost near this equilibrium point, requiring that the second partial derivatives with respect to $s$ of $L_{\mathrm{idm}}$ and $L_{\mathrm{dsm}}$ are equal there. Hence we require

$$
\frac{\partial^{2} L_{\mathrm{dsm}}}{\partial s^{2}} \approx \frac{\partial^{2} L_{\mathrm{idm}}}{\partial s^{2}}
$$

at the equilibrium point $v=v_{\text {eq }}, s=s_{\text {eq }}$. Evaluating the partial derivatives, we find

$$
\frac{\partial^{2} L_{\mathrm{dsm}}}{\partial s^{2}}=\frac{\gamma}{s_{d}^{2}}
$$

and:

$$
\frac{\partial^{2} L_{\mathrm{idm}}}{\partial s^{2}}=\frac{8\left(s^{*}\right)^{4}}{s_{d}^{6}}+\frac{12\left(s^{*}\right)^{2}}{s_{d}^{4}}\left(\frac{\left(s^{*}\right)^{2}}{s_{d}^{2}}+\left(\frac{v}{v_{0}}\right)^{\delta}-1+\frac{u}{a}\right)
$$

To simplify (20), we note that if the DSM is to behave similarly to the IDM near equilibrium, then we will have

$$
\frac{u}{a} \approx 1-\left(\frac{v}{v_{0}}\right)^{\delta}-\frac{\left(s^{*}\right)^{2}}{s_{d}^{2}}
$$

so that the second term may be neglected, leading to:

$$
\frac{\partial^{2} L_{\mathrm{idm}}}{\partial s^{2}}=\frac{8\left(s^{*}\right)^{4}}{s_{d}^{6}}
$$

Recalling that we wished the partial derivatives to be equal at the equilibrium point, we may now equate (19) and (21) and rearrange for $\gamma$ to yield:

$$
\gamma(v)=8\left(\left(v / v_{d}\right)^{\delta}-1\right)^{2}
$$

This completes the specification of the stage cost function for the DSM, as the weighting parameters $\beta$ and $\gamma$ and the desired spacing $s_{d}$ appearing in (11) have now been specified in terms of the driver preferences $a, v_{d}, s_{0}$, and $T$.
Remark 1. If desired, the dependence of $s_{d}$ and $\gamma$ on $v$ may be removed by substitution of $v=v_{L}$ which is true at equilibrium. In that case, the DSM stage cost (11) is quasiconvex in $u$, $v$ and $s$. In particular, this guarantees that it has a single local minimum which is also the global minimum. However, in cases considering limits on speed during cornering as in the following section, the resulting optimisation problem is nonconvex anyway and in such cases the authors have found that retaining the dependence on $v$ typically leads to more natural driving behaviour.

\section{Incorporation of cornering constraints}

So far, the emphasis has been on developing a model compatible with the parameters of the IDM and yielding similar behaviour in vehicle-following. We now extend this to incorporate limits to the speed while cornering, considering the maximum speed as a function of curvature implied by the lateral acceleration limits of [23]:

$$
v \leq \sqrt{\frac{\Gamma_{\max }}{\kappa(x)+\Delta}}
$$

We note that the curvature $\kappa(x)$ depends on the distance travelled down the road, and therefore the constraint function is in general a nonlinear function of travelled distance. Practically, this can be modelled as a cubic spline curve, which is the approach used in our implementation.

For completeness, we now state the entire model:

Optimization 1 (Driver Satisfaction Model). Solve the optimal control problem,

$$
\begin{aligned}
\min _{u(t)} & \int_{0}^{T} L_{d s m}\left(x_{L}-x, v, u\right) d t \\
\text { s.t. } & \dot{x}=v, \quad \dot{v}=u \\
& u \leq a, \quad x_{L}-x \geq 0 \\
& v \leq \sqrt{\Gamma_{\max } /(\kappa(x)+\Delta)} \\
& x(0)=x_{0}, \quad v(0)=v_{0}
\end{aligned}
$$

where the stage cost $L_{d s m}$ is given by

$$
\begin{gathered}
L_{d s m}(s, v, u)=(u / a)^{2}+\delta^{2}\left(v / v_{d}-1\right)^{2} \\
+8\left(\left(v / v_{d}\right)^{\delta}-1\right)^{2} \frac{\left(s / s_{d}-1\right)^{2}}{\left(s / s_{d}\right)^{2}+1}
\end{gathered}
$$

where we have defined

$$
s_{d}=\frac{s_{0}+T v}{\sqrt{1-\left(v_{L} / v_{d}\right)^{\delta}}}
$$

and where $x_{L}$ is the position of the rear of the lead vehicle.

It should be noted that in this form, the model is acausal in that it assumes knowledge of the leader's future position $x_{L}(t)$ and the upcoming road curvature $\kappa(x)$. If it is desired to generate predictions from the model in real time, as is possible for the IDM, some simplifying assumption is required about the future behaviour of the lead vehicle, such as that it continues travelling at its current velocity, accelerates smoothly up to the speed limit, or that it brakes to a halt before an approaching 


\begin{tabular}{l|ll} 
Parameter & Value (IDM) & Value (DSM) \\
\hline$a$ & $4 \mathrm{~m} / \mathrm{s}^{2}$ & $4 \mathrm{~m} / \mathrm{s}^{2}$ \\
$b$ & $4 \mathrm{~m} / \mathrm{s}^{2}$ & - \\
$v_{d}$ & $30 \mathrm{~m} / \mathrm{s}$ & $30 \mathrm{~m} / \mathrm{s}$ \\
$s_{0}$ & $2 \mathrm{~m}$ & $2 \mathrm{~m}$ \\
$T$ & $1.5 \mathrm{~s}$ & $1.5 \mathrm{~s}$ \\
$\delta$ & 4 & 4
\end{tabular}

TABLE I: Model parameters for the comparison
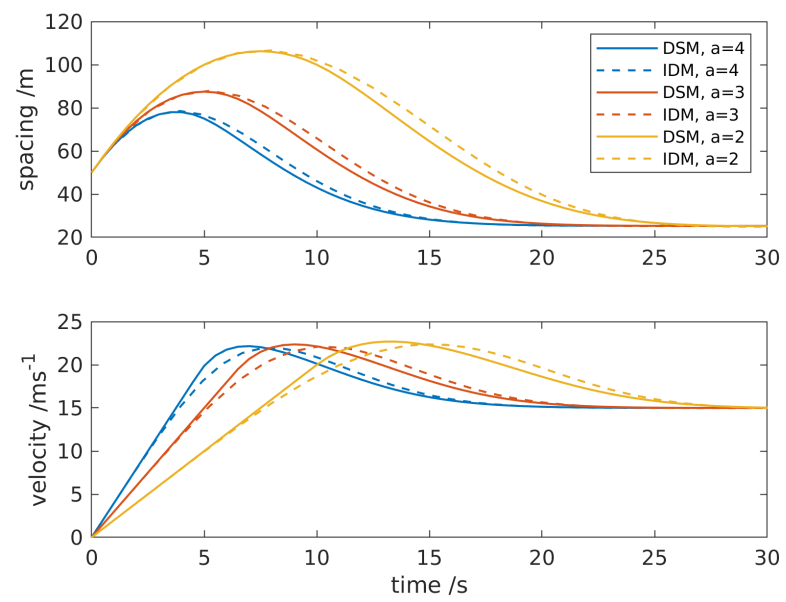

Fig. 1: Car following, comparison with IDM for different ' $a$ '

intersection. When considering curve driving however, acausal modelling is natural in that drivers decelerate in response to upcoming curves, before they are encountered.

\section{Comparison of the model with IDM}

As the DSM expressions for $s_{d}, \beta$ and $\gamma$ developed in the preceding sections were derived to provide similar behaviour to the IDM, it is interesting to compare the predictions of the two models in some common situations. Figure 1 shows a comparison of vehicle velocity under the DSM and the IDM when accelerating from a standing start to follow a vehicle of velocity $15 \mathrm{~m} / \mathrm{s}$, for different values of the parameter $a$. Other parameters used for this comparison are given in Table I. The velocity and spacing of the two models evolve similarly in each case, and increases in $a$ (which represents the driver preference on maximum acceleration) affects both models by increasing the initial acceleration, decreasing the maximum spacing, and increasing the time taken to reach steady state, while leaving the maximum velocity almost unchanged.

One interesting feature of the IDM compared to many other car-following models is the ability to handle emergency braking situations in which the deceleration may be large in order to avoid a collision. As the DSM contains no lower bound on the acceleration and contains a constraint to ensure that the vehicle spacing remains positive, it can also handle harsh braking situations in which large decelerations are required, avoiding a collision if possible to do so. A comparison between the two models under braking is shown in Figure 2, in which the ego vehicle is initially travelling at $30 \mathrm{~m} / \mathrm{s}$ and there is a slower vehicle travelling at $15 \mathrm{~m} / \mathrm{s}$ at $50 \mathrm{~m}$ distance. In this Figure the parameter $T$ is varied, which represents a driver
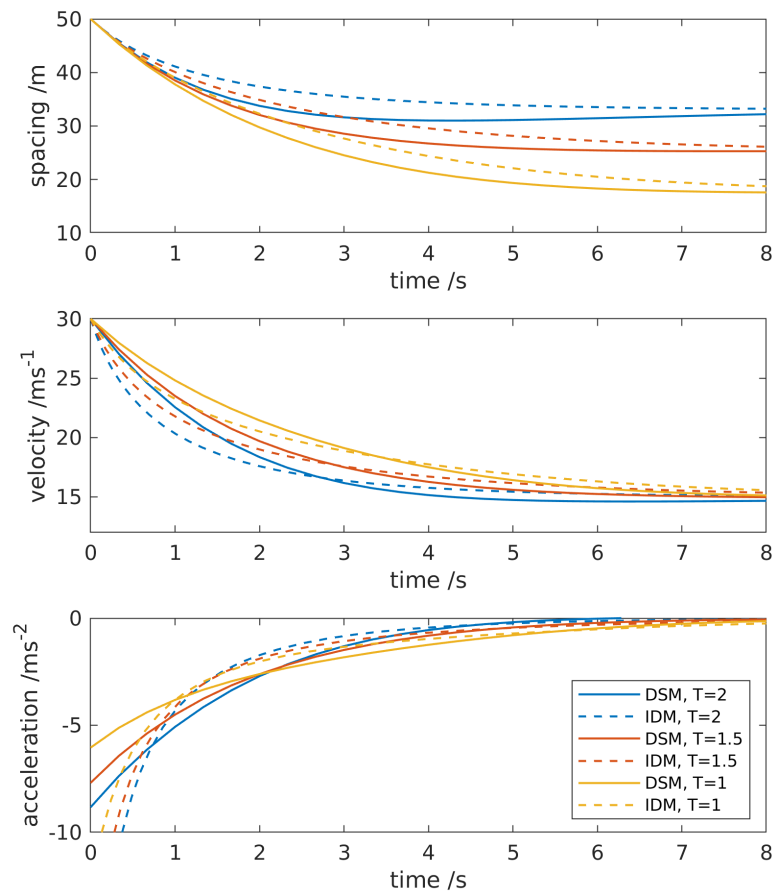

Fig. 2: Braking, comparison with IDM for different ' $T$ '

preference on time headway while following another vehicle. In this case, the final spacing and velocity for the two models is equal, but the IDM shows much greater decelerations initially of over $10 \mathrm{~m} / \mathrm{s}^{2}$. For both models, the effect of increasing $T$ is to increase the final following distance and to increase the initial deceleration, which is expected if a greater final following distance is desired.

\section{Model VALIDATion}

\section{A. Method}

We compared predictions of our model to naturalistic driving data collected by ADAM [43] as part of the G-ACTIVE (Green Adaptive ConTrol for Interconnected VEhicles) project [44]. ADAM is placed in a study participant's own vehicle, and collects data on vehicle position and velocity using GPS as well as the spacing to the lead vehicle by post-processing of recorded stereo video. Data collected from 3 different drivers was used, which included both urban and rural driving situations. To give a fair evaluation of the two models, the data was split into 'training' and 'test' data. The training data consisted of approximately 10 minutes of car-following and cornering data for each driver that were used to fit parameters of the IDM and Reymond's cornering model by minimising the mean square velocity error, as described in [38] and [23]. The parameters of the DSM were then chosen to be identical to the corresponding parameters of the IDM and the cornering model. The test data were seperated into 30 second segments for use as cases for validation. Segments where the driver was travelling at a constant speed were discarded, and the remaining testcases were classified into: starting and stopping, vehicle-following, and cornering, by the following criteria: 

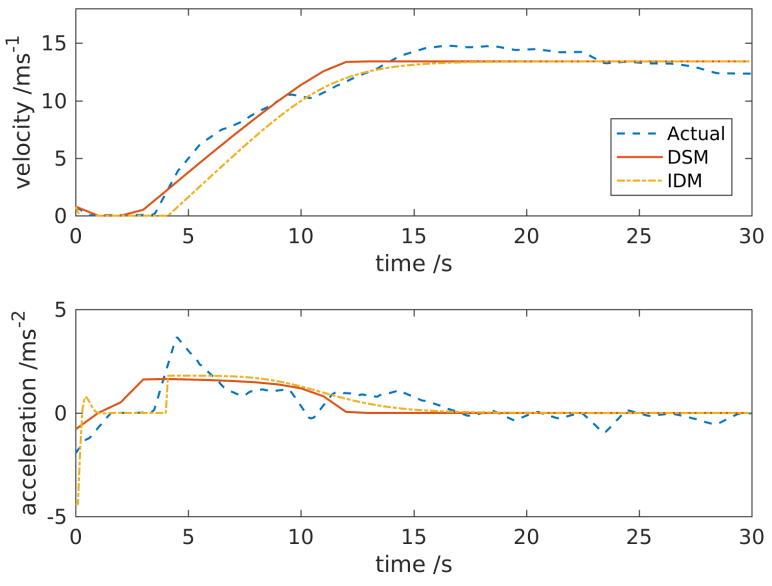

Fig. 3: Example of start/stop testcase (Test S4)
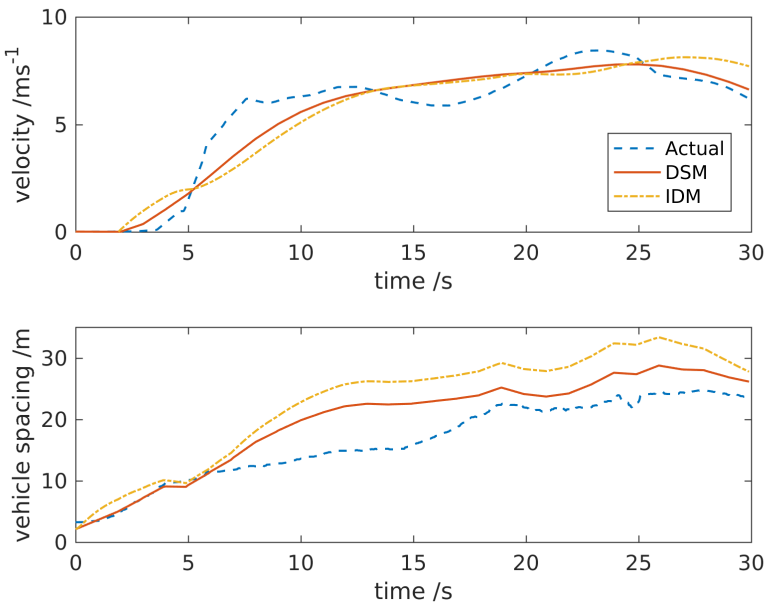

Fig. 4: Example of vehicle-following testcase (Test F4)

- Start/stop: There is no leading vehicle. The driver either brakes to a stop at, or accelerates away from, a static road feature such as an intersection or traffic signals.

- Vehicle-following: The driver is following another vehicle, which is accelerating and braking due to traffic conditions. Corners and intersections may be included.

- Cornering: There is no leading vehicle. The driver's speed varies due to the road curvature at corners and intersections.

For the start/stop and vehicle-following tests, predictions of the IDM are compared to those of the DSM. Similarly, Reymond's model is compared with the predictions of the DSM for the cornering tests. For each testcase, both models were simulated using the leader velocity collected from ADAM and applicable speed limit and road curvature data. We computed the rootmean-square velocity error,

$$
\operatorname{RMSE}=\sqrt{\frac{1}{x(T)} \int_{0}^{x(T)}\left[v_{\text {predicted }}(x)-v_{\text {actual }}(x)\right]^{2} \mathrm{dx}}
$$
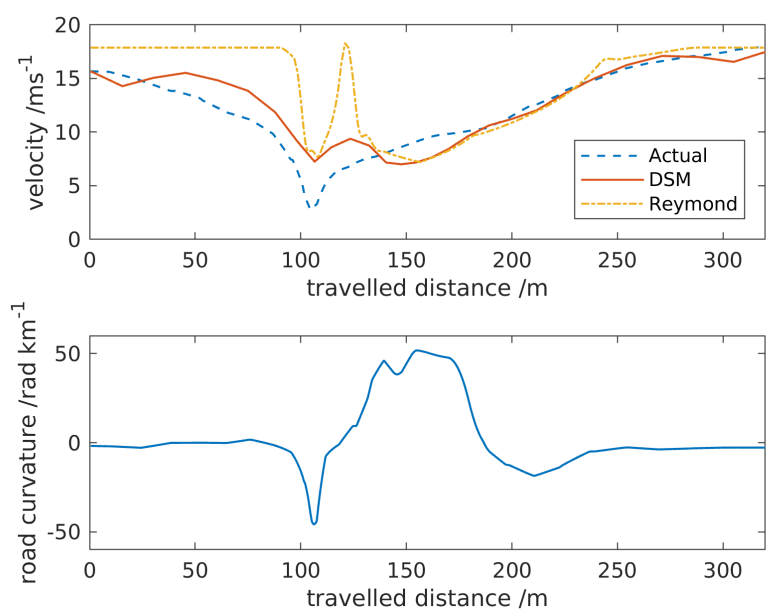

Fig. 5: Example of cornering testcase (Test C4)

\begin{tabular}{|c|c|c|c|c|}
\hline Test & $\begin{array}{l}\mathrm{RMSE}_{\mathrm{DSM}} \\
/ \mathrm{ms}^{-1}\end{array}$ & $\begin{array}{l}\mathrm{RMSE}_{\mathrm{IDM}} \\
/ \mathrm{ms}^{-1}\end{array}$ & $\begin{array}{l}\mathrm{MAXE}_{\mathrm{DSM}} \\
/ \mathrm{ms}^{-1}\end{array}$ & $\begin{array}{l}\text { MAXE }_{\text {IDM }} \\
/ \mathrm{ms}^{-1}\end{array}$ \\
\hline S1 & 1.70 & 0.82 & 3.43 & 2.26 \\
\hline S2 & 0.57 & 0.84 & 1.17 & 2.07 \\
\hline S3 & 2.19 & 1.18 & 4.89 & 3.08 \\
\hline S4 & 0.97 & 1.22 & 1.77 & 3.44 \\
\hline S5 & 0.72 & 1.18 & 1.50 & 2.79 \\
\hline S6 & 0.73 & 1.55 & 1.91 & 4.28 \\
\hline S7 & 2.91 & 2.34 & 6.52 & 5.00 \\
\hline S8 & 3.03 & 2.31 & 7.10 & 4.99 \\
\hline S9 & 2.06 & 2.24 & 4.14 & 3.58 \\
\hline S10 & 1.06 & 2.73 & 2.34 & 4.31 \\
\hline mean & 1.59 & 1.64 & 3.48 & 3.58 \\
\hline corr & & 0.48 & & 0.60 \\
\hline
\end{tabular}

TABLE II: Results for start/stop testcases

and also the maximum velocity error,

$$
\operatorname{MAXE}=\max _{x \in[x(0), x(T)]}\left|v_{\text {predicted }}(x)-v_{\text {actual }}(x)\right|
$$

to measure the goodness-of-fit of the model to the data. We take the integral with respect to distance in the RMS error, rather than with respect to time, to ensure that we are always comparing speeds at the same position on the road. This is especially important for the cornering testcases.

Simulations and the subsequent calculation of error metrics were performed in MATLAB. The DSM was implemented by discretising the model for a step of $1 s$ using direct collocation as described in [32]. The automatic differentiation software CasADi [45] was used to formulate the collocation equations, and the resulting nonlinear programming problem solved with IPOPT [46]. No attempt was made to improve computational efficiency (for example by code generation), yet this solution procedure took an average of $0.42 \mathrm{~s}$ to optimize $30 \mathrm{~s}$ of driver behaviour, using a mobile Intel core i5 processor, indicating that real-time implementation is likely to be feasible.

\section{B. Results and Analysis}

In all three scenarios, the RMSE and MAXE is smaller for the DSM than it is for either the IDM or Reymond's model, although in the car-following and start-stop testcases 


\begin{tabular}{|c|c|c|c|c|}
\hline Test & $\begin{array}{l}\mathrm{RMSE}_{\mathrm{DSM}} \\
/ \mathrm{ms}^{-1}\end{array}$ & $\begin{array}{l}\mathrm{RMSE}_{\mathrm{IDM}} \\
/ \mathrm{ms}^{-1}\end{array}$ & $\begin{array}{l}\text { MAXE }_{\text {DSM }} \\
/ \mathrm{ms}^{-1}\end{array}$ & $\begin{array}{l}\text { MAXE IDM } \\
/ \mathrm{ms}^{-1}\end{array}$ \\
\hline F1 & 0.67 & 0.81 & 1.33 & 1.70 \\
\hline F2 & 1.31 & 0.65 & 1.87 & 3.70 \\
\hline F3 & 1.48 & 1.51 & 2.65 & 2.46 \\
\hline F4 & 0.82 & 1.11 & 2.08 & 2.70 \\
\hline F5 & 0.55 & 0.48 & 1.38 & 1.38 \\
\hline F6 & 0.30 & 0.32 & 0.71 & 0.87 \\
\hline F7 & 0.59 & 0.69 & 0.94 & 1.05 \\
\hline F8 & 0.34 & 0.47 & 0.81 & 1.01 \\
\hline F9 & 0.45 & 0.43 & 0.73 & 0.92 \\
\hline F10 & 1.48 & 2.80 & 3.11 & 4.34 \\
\hline mean & 0.80 & 0.93 & 1.56 & 2.01 \\
\hline corr & & 0.78 & & 0.89 \\
\hline
\end{tabular}

TABLE III: Results for vehicle-following testcases

\begin{tabular}{l|ll|ll} 
Test & $\begin{array}{l}\mathrm{RMSE}_{\mathrm{DSM}} \\
\mathrm{ms}^{-1}\end{array}$ & $\begin{array}{l}\mathrm{RMSE}_{\text {Rey }} \\
/ \mathrm{ms}^{-1}\end{array}$ & $\begin{array}{l}\mathrm{MAXE}_{\mathrm{DSM}} \\
/ \mathrm{ms}^{-1}\end{array}$ & $\begin{array}{l}\mathrm{MAXE}_{\text {Rey }} \\
/ \mathrm{ms}^{-1}\end{array}$ \\
\hline C1 & 3.94 & 4.21 & 6.47 & 8.95 \\
C2 & 2.74 & 4.93 & 4.11 & 9.17 \\
C3 & 2.23 & 3.28 & 5.19 & 7.03 \\
C4 & 1.84 & 3.68 & 5.99 & 10.34 \\
C5 & 1.60 & 2.39 & 4.75 & 9.39 \\
C6 & 2.90 & 3.61 & 8.83 & 12.85 \\
C7 & 5.13 & 3.56 & 8.21 & 16.20 \\
C8 & 1.07 & 1.51 & 2.83 & 4.10 \\
C9 & 5.38 & 5.49 & 15.05 & 15.05 \\
C10 & 3.08 & 6.35 & 4.65 & 12.35 \\
\hline mean & 2.99 & 3.90 & 6.61 & 10.54 \\
corr & & 0.58 & & 0.73
\end{tabular}

TABLE IV: Results for cornering testcases

this improvement appears minor. All scenarios show a positive correlation between the error metric when simulating using the DSM and when using the reference model, implying that the DSM tends to perform well for the testcases in which the reference models perform well, and vice versa.

To determine if these reductions in error were statistically significant, we compared the RMSE values for the DSM with that of the benchmark using the Wilcoxon signed-rank test, a non-parametric test of the paired differences. This indicated that the differences in RMSE for the start/stop $(Z=0, p=1$, $r=0)$ and vehicle-following $(Z=-1.27, p=0.202$, $r=0.40$ ) testcases were not significant at the 0.05 significance level. For the cornering testcase, the differences in RMSE were significant $(Z=-2.08, p=0.037, r=0.66)$, which was also the case for the MAXE error metric $(Z=-2.61$, $p=0.009, r=0.82$ ). This suggests that the DSM gives comparable performance to the IDM in car-following and startstop situations, while outperforming the model of Reymond et al. in cornering situations.

\section{USAGE EXAMPLE}

We now demonstrate the use of the DSM in an eco-driving optimization by considering a braking scenario in which an electric vehicle must slow from highway speeds to a low speed due to a corner, as may occur at the end of an offramp or sliproad. We consider a vehicle that may regenerate a factor $0 \leq \theta \leq 1$ of braking energy, and has energy losses
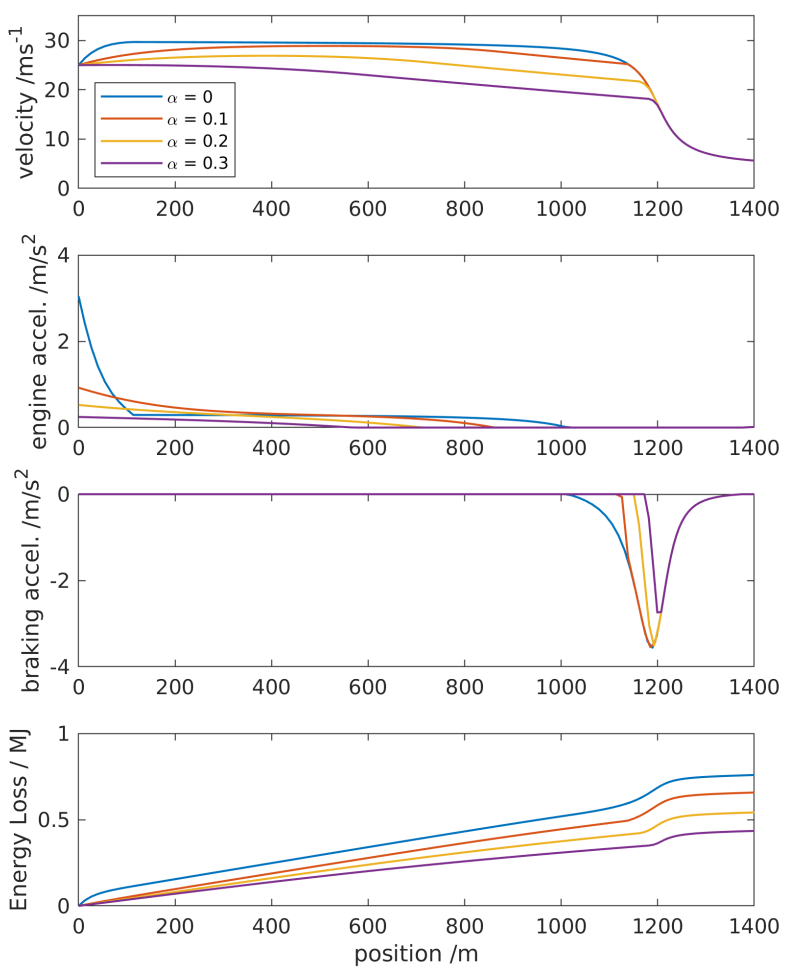

Fig. 6: Eco-driving optimal control example

due to aerodynamic drag and rolling resistance in which the combined drag force is given by

$$
F_{\text {drag }}=c_{d} v^{2}+m c_{r r}=\frac{1}{2} \rho_{\text {air }} C_{D} A v^{2}+C_{R R} m g
$$

in which $C_{D} A$ and $C_{R R}$ denotes the usual coefficients of aerodynamic drag and rolling resistance, and $m g$ is the weight of the vehicle. We additionally consider a simplified model of an electric powertrain with losses due to the resistance $R_{m}$ of the motor windings, and a reduction gear of ratio $N_{g}$. These resistive losses are given by $R_{m} i_{m}^{2}$ where $i_{m}$ is the motor current given by

$$
i_{m}=\frac{r F}{N_{g} k}
$$

in which $F$ denotes the traction force at the wheels, $r$ denotes wheel radius, and $k$ is the torque constant of the electrical motor. For simplicity, we ignore other electrical losses.

To correctly handle the energy loss when braking, we introduce different input variables for acceleration due to the vehicle engine and brakes, considering $u=u_{e}+u_{b}$ where $u_{e} \geq 0$ is the component of acceleration due to the engine only, and $u_{b} \leq 0$ is that due to braking. Considering the $u$ term in (11), we can expand in $u_{e}$ and $u_{b}$ giving

$$
(u / a)^{2}=\left(u_{e} / a\right)^{2}+2 u_{e} u_{b} / a^{2}+\left(u_{b} / a\right)^{2}
$$

and noting that $u_{e} u_{b}=0$ if, as in normal driving, the accelerator and brake are not used simultaneously, we find:

$$
(u / a)^{2}=\left(u_{e} / a\right)^{2}+\left(u_{b} / a\right)^{2}
$$


In fact, as we may always establish this equality for any solution $u(t)$ of Optimization 1 by choosing $u_{e}(t)=\max (0, u(t))$ and $u_{b}(t)=\min (0, u(t))$, it is evident that using the expression on the right hand side of (24) in place of $(u / a)^{2}$ in the DSM stage cost will ensure that accelerator and brake are not activated simultaneously, without otherwise changing the result. This leads to the modified stage cost function:

$$
\begin{aligned}
L_{\mathrm{dsm}}^{\prime} & =\left(u_{e} / a\right)^{2}+\left(u_{b} / a\right)^{2}+\delta^{2}\left(v / v_{d}-1\right)^{2} \\
& +8\left(\left(v / v_{d}\right)^{\delta}-1\right)^{2} \frac{\left(s / s_{d}-1\right)^{2}}{\left(s / s_{d}\right)^{2}+1}
\end{aligned}
$$

For completeness, we state the optimal control problem with this modified stage cost.

Optimization 2 (Example eco-driving optimization). Solve the optimal control problem,

$$
\begin{aligned}
\min _{u(t)} & \int_{0}^{T}\left[L_{d s m}^{\prime}\left(x_{L}-x, v, u_{e}, u_{b}\right)+\alpha L_{\text {loss }}(v)\right] d t \\
\text { s.t. } & \dot{x}=v, \quad \dot{v}=u_{e}+u_{b}-c_{d} v^{2}-c_{r r} \\
& 0 \leq u_{e} \leq a, \quad u_{b} \leq 0, \quad s, v \geq 0 \\
& v \leq \sqrt{\Gamma_{\max } /(\kappa(x)+\Delta)} \\
& x(0)=x_{0}, \quad v(0)=v_{0}
\end{aligned}
$$

where the DSM stage cost $L_{d s m}^{\prime}$ is given by

$$
\begin{aligned}
L_{d s m}^{\prime} & =\left(u_{e} / a\right)^{2}+\left(u_{b} / a\right)^{2}+\delta^{2}\left(v / v_{d}-1\right)^{2} \\
& +8\left(\left(v_{L} / v_{d}\right)^{\delta}-1\right)^{2} \frac{\left(s / s_{d}-1\right)^{2}}{\left(s / s_{d}\right)^{2}+1}
\end{aligned}
$$

with $s_{d}(v)$ is defined as in Optimization 1, and

$$
L_{\text {loss }}(v)=-(1-\theta) m u_{b} v+c_{d} v^{3}+c_{r r} v+R_{m}\left(\frac{r m u_{e}}{N_{g} k}\right)^{2}
$$

is the stage cost associated with energy losses.

The result of this optimization for different values of $\alpha$ in the braking scenario is shown in Figure 6. The corner is modelled as a cubic spline of increasing curvature as a function of $x$, with curvature increasing over an approximately $80 \mathrm{~m}$ length, and a final (maximum) curvature chosen to correspond to a cornering speed of $6 \mathrm{~m} / \mathrm{s}$. The initial velocity was taken as $v_{0}=25 \mathrm{~m} / \mathrm{s}$, and the vehicle is initially at $x_{0}=0 \mathrm{~km}$ with the corner placed at $x_{c}=1.2 \mathrm{~km}$. For the vehicle, we take $m=$ $1500 \mathrm{~kg}, r=0.29, C_{D} A=0.7$ and $C_{R R}=0.005$, typical for a medium-sized family car. For the electric powertrain we set $k=0.12, R_{m}=0.1, N_{g}=15$, and $\theta=0.7$. The driver preference parameters used in the DSM are those in Table I that were used for the previous comparison examples.

It is apparent that as the parameter $\alpha$ penalising energy usage is increased, the electric motor is used less and at lower torques on approach to the corner, with a greater reduction in speed via coasting under the effects of drag. In the most extreme case of $\alpha=0.3$, this requires $900 \mathrm{~m}$ of coasting and reduces energy loss by approximately $43 \%$, but intermediate values of $\alpha$ also give reductions in energy loss while remaining closer to the 'natural' velocity profile given by the $\alpha=0$ case.

\section{CONCLUSION}

We have introduced a method based on optimal control model to incorporate driver preferences into eco-driving assistance systems. Models of longitudinal driver behaviour available from the traffic modelling literature were unsuitable for this purpose, so we developed a novel Driver Satisfaction Model that characterises the driver using parameters in common with the well-known Intelligent Driver Model, but in addition is compatible with eco-driving optimal control problems and is capable of reproducing driver speed in curves. As the DSM is based on optimal control, it may be directly used for eco-driving optimizations in which fuel-saving velocity profiles are sought while respecting a 'natural' driving style. The model has been validated by comparison with naturalistic driving data in several scenarios, finding it gives comparable or better performance than existing models in the literature for prediction of the speed of an individual vehicle in typical vehicle-following and cornering situations. Finally, we illustrated the potential for optimal control to be used to tradeoff energy savings and a natural driving style in a braking and cornering scenario for an electric vehicle.

\section{ACKNOWLEDGEMENT}

We are grateful for support of the UK Engineering and Physical Sciences Research Council, grant number EP/N022262/1 (Green Adaptive Control for Future Interconnected Vehicles).

\section{REFERENCES}

[1] L. Evans, "Driver behavior effects on fuel consumption in urban driving," in Proceedings of the Human Factors Society Annual Meeting, vol. 22, no. 1. Sage Publications Sage CA: Los Angeles, CA, 1978, pp. 437-442.

[2] M. P. Vandenbergh, J. Barkenbus, and J. Gilligan, "Individual carbon emissions: The low-hanging fruit," UCLA L. Rev., vol. 55, p. 1701, 2007.

[3] J. N. Barkenbus, "Eco-driving: An overlooked climate change initiative," Energy Policy, vol. 38, no. 2, pp. 762-769, 2010.

[4] V. Heijne, N. Ligterink, and U. Stelwagen, "Potential of eco-driving. UDRIVE Deliverable D45.1. EU FP7 Project UDRIVE Consortium. https://doi.org/10.26323/UDRIVE_D45.1," 2017.

[5] M. Zarkadoula, G. Zoidis, and E. Tritopoulou, "Training urban bus drivers to promote smart driving: A note on a Greek eco-driving pilot program," Transportation Research Part D: Transport and Environment, vol. 12, no. 6, pp. 449-451, 2007.

[6] B. Beusen, S. Broekx, T. Denys, C. Beckx, B. Degraeuwe, M. Gijsbers, K. Scheepers, L. Govaerts, R. Torfs, and L. I. Panis, "Using on-board logging devices to study the longer-term impact of an eco-driving course," Transportation research part D: transport and environment, vol. 14, no. 7, pp. 514-520, 2009.

[7] H. Rakha and R. K. Kamalanathsharma, "Eco-driving at signalized intersections using V2I communication," in Intelligent Transportation Systems (ITSC), 2011 14th International IEEE Conference on. IEEE, 2011, pp. 341-346.

[8] S. Azzi, G. Reymond, F. Mérienne, and A. Kemeny, "Eco-driving performance assessment with in-car visual and haptic feedback assistance," Journal of Computing and Information Science in Engineering, vol. 11, no. 4, p. 041005, 2011.

[9] S. A. Birrell, M. S. Young, and A. M. Weldon, "Vibrotactile pedals: provision of haptic feedback to support economical driving," Ergonomics, vol. 56, no. 2, pp. 282-292, 2013.

[10] S. E. Li, S. Xu, X. Huang, B. Cheng, and H. Peng, "Eco-departure of connected vehicles with v2x communication at signalized intersections," IEEE Transactions on Vehicular Technology, vol. 64, no. 12, pp. 54395449, 2015. 
[11] H. Ma, H. Xie, and D. Brown, "Eco-driving assistance system for a manual transmission bus based on machine learning," IEEE Transactions on Intelligent Transportation Systems, vol. 19, no. 2, pp. 572-581, 2018.

[12] M. Wang, W. Daamen, S. P. Hoogendoorn, and B. van Arem, "Rolling horizon control framework for driver assistance systems. part i: Mathematical formulation and non-cooperative systems," Transportation research part C: emerging technologies, vol. 40, pp. 271-289, 2014.

[13] A. Sciarretta, G. De Nunzio, and L. L. Ojeda, "Optimal ecodriving control: Energy-efficient driving of road vehicles as an optimal control problem," IEEE Control Systems, vol. 35, no. 5, pp. 71-90, 2015.

[14] Y. Saboohi and H. Farzaneh, "Model for developing an eco-driving strategy of a passenger vehicle based on the least fuel consumption," Applied Energy, vol. 86, no. 10, pp. 1925-1932, 2009.

[15] F. Mensing, R. Trigui, and E. Bideaux, "Vehicle trajectory optimization for application in eco-driving," in Vehicle Power and Propulsion Conference (VPPC), 2011 IEEE. IEEE, 2011, pp. 1-6.

[16] M. A. S. Kamal, M. Mukai, J. Murata, and T. Kawabe, "On board eco-driving system for varying road-traffic environments using model predictive control." in $C C A, 2010$, pp. 1636-1641.

[17] Q. Jin, G. Wu, K. Boriboonsomsin, and M. J. Barth, "Power-based optimal longitudinal control for a connected eco-driving system," IEEE Transactions on Intelligent Transportation Systems, vol. 17, no. 10, pp. 2900-2910, 2016

[18] F. Ding and H. Jin, "On the optimal speed profile for eco-driving on curved roads," IEEE Transactions on Intelligent Transportation Systems, vol. 19, no. 12, pp. 4000-4010, 2018.

[19] R. Parasuraman, P. A. Hancock, and O. Olofinboba, "Alarm effectiveness in driver-centred collision-warning systems," Ergonomics, vol. 40, no. 3, pp. 390-399, 1997

[20] J. M. Fleming, C. K. Allison, X. Yan, N. A. Stanton, and R. Lot, "Adaptive driver modelling in ADAS to improve user acceptance: A study using naturalistic data," Safety Science, 2018.

[21] X. Xiang, K. Zhou, W.-B. Zhang, W. Qin, and Q. Mao, "A closedloop speed advisory model with driver's behavior adaptability for eco-driving," IEEE Transactions on Intelligent Transportation Systems, vol. 16, no. 6, pp. 3313-3324, 2015.

[22] M. Treiber, A. Hennecke, and D. Helbing, "Congested traffic states in empirical observations and microscopic simulations," Physical review E, vol. 62 , no. 2 , p. 1805,2000

[23] G. Reymond, A. Kemeny, J. Droulez, and A. Berthoz, "Role of lateral acceleration in curve driving: Driver model and experiments on a real vehicle and a driving simulator," Human factors, vol. 43, no. 3, pp. 483-495, 2001.

[24] J. Monteil, N. OHara, V. Cahill, and M. Bouroche, "Real-time estimation of drivers' behaviour," in Intelligent Transportation Systems (ITSC), 2015 IEEE 18th International Conference on. IEEE, 2015, pp. 20462052.

[25] J. M. Fleming, X. Yan, and R. Lot, "Fitting cornering speed models with one-class support vector machines," in 2019 IEEE Intelligent Vehicles Symposium (IV). IEEE, 2019, pp. 2457-2462.

[26] R. E. Bellman, "Dynamic programming," 1957.

[27] L. S. Pontryagin, E. Mishchenko, V. Boltyanskii, and R. Gamkrelidze, "The mathematical theory of optimal processes," 1962.

[28] J. Fleming, X. Yan, C. Allison, N. Stanton, and R. Lot, "Driver modeling and implementation of a fuel-saving ADAS," in IEEE Conference on Systems, Man and Cybernetics, 2018.

[29] R. Bellman, I. Glicksberg, and O. Gross, "On the bang-bang control problem," Quarterly of Applied Mathematics, vol. 14, no. 1, pp. 11-18, 1956.

[30] R. E. Kalman, "Contributions to the theory of optimal control," Bol. Soc. Mat. Mexicana, vol. 5, no. 2, pp. 102-119, 1960.

[31] H. G. Bock and K.-J. Plitt, "A multiple shooting algorithm for direct solution of optimal control problems," IFAC Proceedings Volumes, vol. 17 , no. 2, pp. 1603-1608, 1984.

[32] C. R. Hargraves and S. W. Paris, "Direct trajectory optimization using nonlinear programming and collocation," Journal of Guidance, Control, and Dynamics, vol. 10, no. 4, pp. 338-342, 1987.

[33] R. E. Chandler, R. Herman, and E. W. Montroll, "Traffic dynamics: studies in car following," Operations research, vol. 6, no. 2, pp. 165184,1958

[34] P. G. Gipps, "A behavioural car-following model for computer simulation," Transportation Research Part B: Methodological, vol. 15, no. 2, pp. 105-111, 1981.

[35] M. Bando, K. Hasebe, A. Nakayama, A. Shibata, and Y. Sugiyama, "Dynamical model of traffic congestion and numerical simulation," Physical review E, vol. 51, no. 2, p. 1035, 1995.
[36] S. Krauß, P. Wagner, and C. Gawron, "Metastable states in a microscopic model of traffic flow," Physical Review E, vol. 55, no. 5, p. 5597, 1997.

[37] R. E. Wilson and J. A. Ward, "Car-following models: fifty years of linear stability analysis-a mathematical perspective," Transportation Planning and Technology, vol. 34, no. 1, pp. 3-18, 2011.

[38] A. Kesting and M. Treiber, "Calibrating car-following models by using trajectory data: Methodological study," Transportation Research Record: Journal of the Transportation Research Board, no. 2088, pp. 148-156, 2008.

[39] G. Burnham, J. Seo, and G. Bekey, "Identification of human driver models in car following," IEEE transactions on Automatic Control, vol. 19, no. 6, pp. 911-915, 1974.

[40] H. Godthelp, "Vehicle control during curve driving," Human Factors, vol. 28, no. 2, pp. 211-221, 1986

[41] W. Van Winsum and H. Godthelp, "Speed choice and steering behavior in curve driving," Human factors, vol. 38, no. 3, pp. 434-441, 1996.

[42] P. Bosetti, M. Da Lio, and A. Saroldi, "On curve negotiation: From driver support to automation," IEEE Transactions on Intelligent Transportation Systems, vol. 16, no. 4, pp. 2082-2093, 2015.

[43] X. Yan, J. Fleming, C. Allison, and R. Lot, "Portable Automobile Data Acquisition Module (ADAM) for naturalistic driving study," in 15th European Automotive Congress (EAEC 2017), 2017.

[44] R. Lot and X. Yan, "The G-ACTIVE project," http://www.g-active.uk, 2016, accessed: 2018-04-13.

[45] J. Andersson, J. Åkesson, and M. Diehl, "Casadi: A symbolic package for automatic differentiation and optimal control," in Recent advances in algorithmic differentiation. Springer, 2012, pp. 297-307.

[46] A. Wächter and L. T. Biegler, "On the implementation of an interiorpoint filter line-search algorithm for large-scale nonlinear programming," Mathematical programming, vol. 106, no. 1, pp. 25-57, 2006.

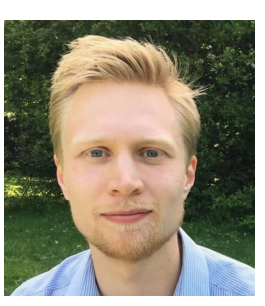

James Fleming (M'12) was born in County Durham, UK in 1990. He received the MEng and DPhil degrees in Engineering Science from the University of Oxford, UK in 2012 and 2016 respectively.

In 2016, he joined the Faculty of Engineering and Physical Sciences at the University of Southampton as a Research Fellow to work on the G-Active (Green, Adaptive Control of Interconnected Vehicles) project. Since September 2019, he has been a Lecturer in the Wolfson School of Electrical, Mechanical and Manufacturing Engineering at Loughborough University. $\mathrm{He}$ researches the theory and practice of optimal and model predictive control, including applications to fuel-efficient driving, motorcycle stabilisation, and renewable energy.

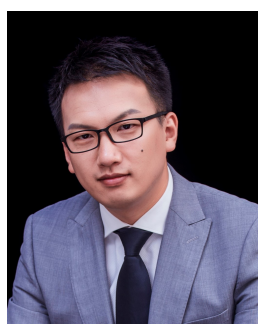

Xingda Yan was born in 1989. He received the B.Eng. degree in automation from Harbin Institute of Technology, Harbin, China in 2012, and the Ph.D. degree in electrical engineering from the University of Southampton, U.K in 2017.

He worked as a Research Fellow with the Faculty of Engineering and Physical Sciences at the University of Southampton from 2016 to 2019 . He is currently a research fellow in the Department of Mechanical Engineering Sciences, University of Surrey, U.K. His research interests include power electronics, hybrid system modelling and control, model predictive control, hybrid electric vehicle modelling and energy management.

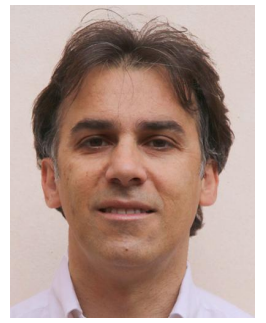

Roberto Lot received a Master's Degree cum laude in Mechanical Engineering in 1994 and a PhD in Mechanics of Machines in 1998 from the University of Padova, Italy.

From 2014 to 2019 he was Professor of Automotive Engineering at the University of Southampton, UK. Currently he is within the Department of Industrial Engineering at the University of Padova. His research interests include dynamics and control of road and race vehicles and contributions to make our vehicles safer, faster, and more eco-friendly. $\mathrm{He}$ has directed several national and international research projects and published more than 100 scientific papers. 